\title{
Four-Way Power Combining Integrated Antenna
}

\author{
Kimberley W. Eccleston \\ Dept of Electrical and Computer Engineering, University of Canterbury, \\ Christchurch, New Zealand
}

Tel: +64-3-364 2987 ext 7045, Fax: +64-3-364-2761, E-mail: kim.eccleston@canterbury.ac.nz

\begin{abstract}
In this paper we show an edge-fed 4port microstripline integrated antenna that has the dual function of power combining as well as being a radiator. The radiation pattern is similar to a two element microstrip half-wave patch array. The edge-feeding allows transistors of an amplifier to be connected directly to the antenna thereby eliminating interconnecting lines. The antenna provides ease of transistor biasing without the need for extra bias circuitry. Experimental results have validated simulations for a prototype operating at 2.47 GHz.
\end{abstract}

Index Terms - Active integrated antennas, microstripline antennas, phased arrays.

\section{INTRODUCTION}

A microstrip antenna can easily be integrated with microstrip based circuits on the same substrate. Moreover, the antenna can be incorporated into the circuit to serve multiple roles other than merely being a radiator [1]. When incorporated into an active circuit, it is called an active integrated antenna [1]. Because such antenna can have multiple roles, other components and interconnecting transmission lines are eliminated thereby reducing size and loss.

A microwave solid-state transmitter may use power combining techniques to achieve sufficient power levels for transmission, and typically comprises several power amplifiers, power combiners, and finally an antenna to radiate the power. It has been demonstrated that the integrated antenna can also be used to combine power [2] - [6]. Most realizations, however, have only considered 2-way power combining [2] - [5]. The author has recently proposed a 4-port microstrip patch integrated antenna that allowed 4-way combining [6]. However, the bandwidth was very narrow, and the port impedance is high. In this work we consider a modified version of the 4-port microstrip antenna that has increased bandwidth, lowered port impedance and has an improved radiation pattern.

\section{OVERVIEW OF THE PROPOSED 4-PORT ANTENNA}

We are interested in integrating a microstrip antenna into the dual-fed distributed amplifier (DFDA), as such amplifiers inherently integrate efficient power combining with amplification [7]. A candidate antenna needs to have its ports running along one edge of the patch and the proposed antenna fulfilling this requirement is shown in Fig 1 along with a schematic showing how it is integrated with the DFDA.

(a)

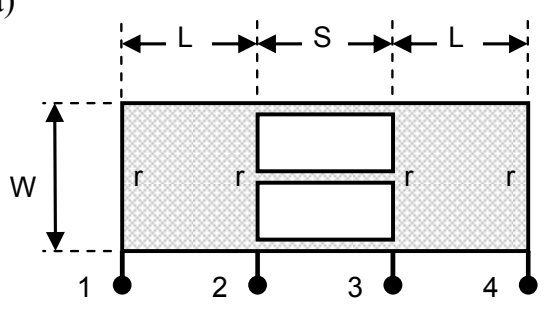

(b)

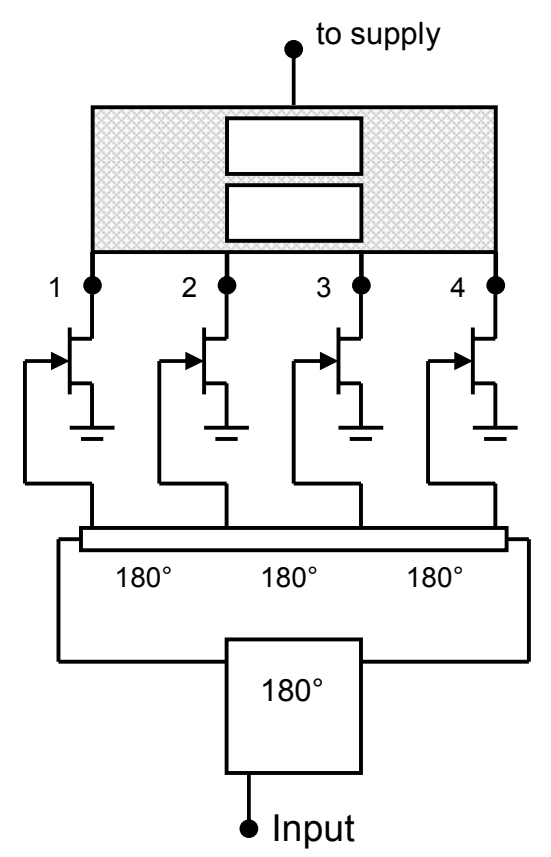

Fig. 1. Proposed 4-port power combining antenna: (a) details of antenna including radiating edges indicated with an " $r$ ", and (b) integration of antenna with transistors to form a DFDA. 
With reference to Fig 1(a), the antenna comprises a pair of patches whose widths are W. The lengths, $\mathrm{L}$, and separation, $\mathrm{S}$, are approximately $\lambda_{\mathrm{g}} / 2$. The two patches are connected together by three narrow microstriplines. When each port is given the same level of excitation, but with alternating polarity, the antenna is resonant in the length-wise direction. Under this condition, the antenna radiates along edges shown (r). Such an excitation can be provided by the dual-fed distributed amplifier technique [7] as shown in Fig 1(b). Because of this resonance mode, midpoint along the narrow microstriplines is effectively short-circuited and this allows convenient application of the drain bias voltage as shown in Fig 1(b). The length of each radiating edge is either $\mathrm{W}$ or approximately $\mathrm{W}$.

\section{COMPARISON WITH OTHER 4-PORT MICROSTRIP ANTENNAS}

It is useful to compare the proposed 4-port antenna (Fig 1) with the previous 4-port antenna [6] shown in Fig 2(a) and a pair of patches Fig 2(b). In both cases, and similar to the proposed antenna (Fig 1(a)), the ports have the same level of excitation but with alternating polarity and this establishes a resonance mode in the length-wise direction of $3 \lambda_{\mathrm{g}} / 2$ for the previous 4 -port antenna (Fig 2(a)) and $\lambda_{\mathrm{g}} / 2$ for the pair of patches (Fig 2(b)). The excitation of the patches in Fig 2(b) is similar to that of other active integrated antennas [2] - [5].

The previous 4-port antenna [6] has two radiating edges of length $\mathrm{W}$ that need to be shared amongst four transistors. In the case of the proposed antenna (Fig 1) and the pair of patches (Fig 2(b)), there are four radiating edges - or one for each transistor. We know that the radiation conductance increases with $\mathrm{W}$ [8], hence the previous 4-port antenna needs to be significantly wider than the proposed 4-port antenna to achieve a radiation conductance per transistor equal to that of the proposed antenna.

The two radiating edges for the previous 4port antenna are separated by $3 \lambda_{\mathrm{g}} / 2$ and means that there will be multiple lobes in the radiation pattern. On the other hand, the proposed 4-port antenna and the pair of patches comprise radiating edges separated by about $\lambda_{\mathrm{g}} / 2$, resulting in a distinct main lobe.

The proposed antenna allows drain bias to be supplied from one bias circuit whereas the pair of patches would require two bias circuits since the patches are isolated at dc.

Method of moment simulations of the antennas were performed using Sonnet ${ }^{1}$. The substrate considered has a dielectric constant of 2.22 and a thickness of $0.7874 \mathrm{~mm}$. The parameters were: $\mathrm{W}$ $=72 \mathrm{~mm}$ (all three antennas), $\mathrm{L}=40 \mathrm{~mm}$ and $\mathrm{S}=$ $38 \mathrm{~mm}$ (for Fig 1(a), and Fig 2(b)), and L' = 118 $\mathrm{mm}$ (for Fig 2(a)). For the proposed antenna, the width of the three microstrips connecting the two patches was $2 \mathrm{~mm}$. In all cases, the width of the ports was $1 \mathrm{~mm}$. The analysis domain (box) size was $600 \mathrm{~mm}$ by $600 \mathrm{~mm}$ and the grid size was 1 $\mathrm{mm}$ by $1 \mathrm{~mm}$. In the case of the proposed antenna, the dc feed port was replaced with a short-circuit to ground.

(a)

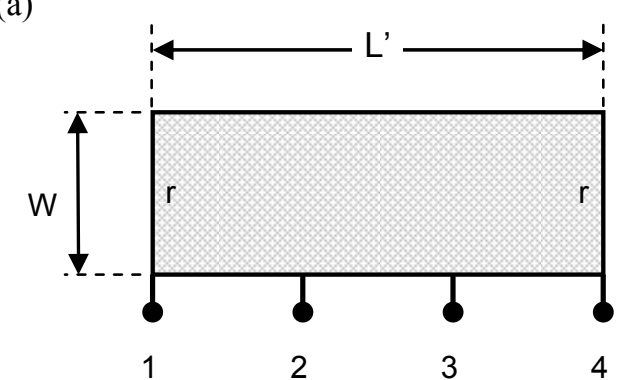

(b)

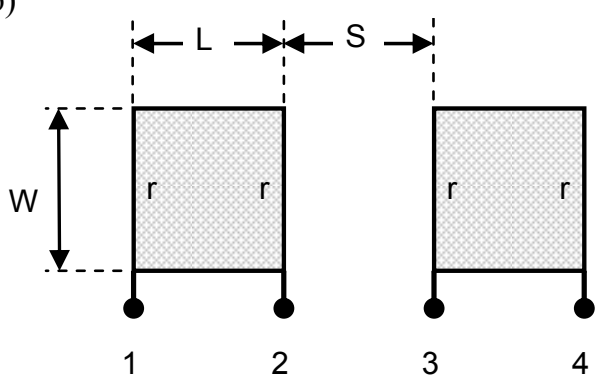

Fig. 2. Other four-port antennas: (a) previous 4-port integrated antenna [6], and (b) array of 2-port patches. Radiating edges are indicated with an " $r$ ".

As the antennas are to be driven by transistors ideally current sources - it is of interest to examine the driving point $Z$-parameters $Z_{\mathrm{kk}}$ for all three antennas. Due to symmetry, only $Z_{11}$ and $Z_{22}$ are shown in Fig 3. It can be seen that for the proposed antenna, $Z_{11}$ and $Z_{22}$ are similar which means that all transistor drive into a similar load which is important for efficiency and equal utilization of transistors [7]. The average radiation resistance per transistor is $272 \Omega$. In the case of the previous 4-port antenna, the radiation resistance per transistor is $508 \Omega$, but there is clearly better similarity between $Z_{11}$ and $Z_{22}$. In the case of the pair of patches, $Z_{11}$ and $Z_{22}$ are essentially equal (as expected) and the radiation resistance per transistor is $218 \Omega$. These values of radiation resistance are consistent with approximate expressions for radiation resistance

\footnotetext{
${ }^{1}$ Sonnet is a trademark of Sonnet Software Inc: www.sonnetsoftware.com
} 
[8]. The bandwidths of the proposed antenna is 1 $\%$, whilst that of the previously proposed antenna is only $0.4 \%$, and that of the pair of patches is 1.3 $\%$.

(a)

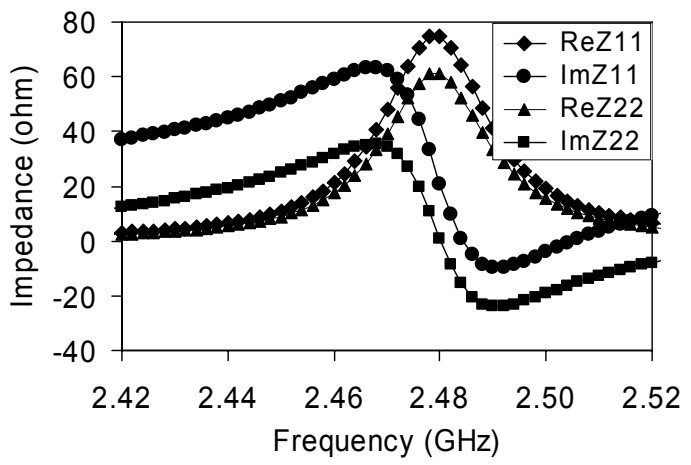

(b)

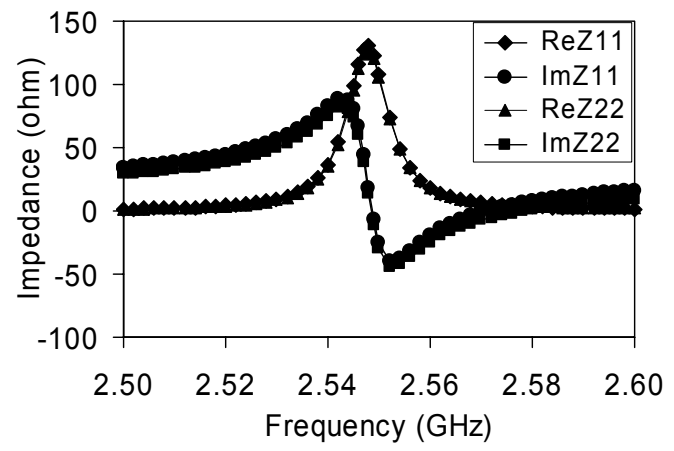

(c)

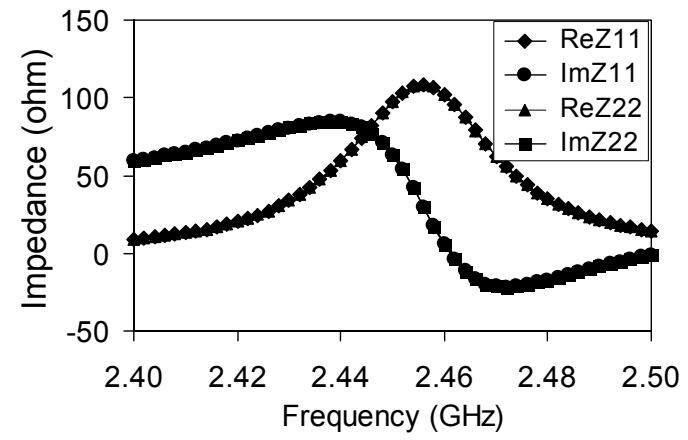

Fig. 3. Calculated $Z_{11}$ and $Z_{22}$ frequency responses of: (a) proposed antenna, (b) previous 4-port antenna [6] and (c) pair of patches.

Fig 4 shows the E-plane radiation patterns for both the proposed antenna and the pair of patches at the centre frequencies of $2.482 \mathrm{GHz}$ and 2.46 $\mathrm{GHz}$ respectively. The radiation patterns are very similar though the side-lobe level of the proposed antenna is slightly higher. The radiation pattern of the previous 4-port antenna is distinctly different [6].

Fig 5 shows the calculated current distributions when only port 1 or port 2 of the proposed antenna is excited (and with the same amplitude). As can be seen, excitation to either port establishes identical resonance modes with similar current densities. This demonstrates that 4-way power combining takes place on the antenna. In the case of the pair of patches, each patch provides two way combining [2] and 4-way combining occurs in space. Therefore, the proposed 4-port antenna would be more tolerant to transistor mismatches than the pair of patches.

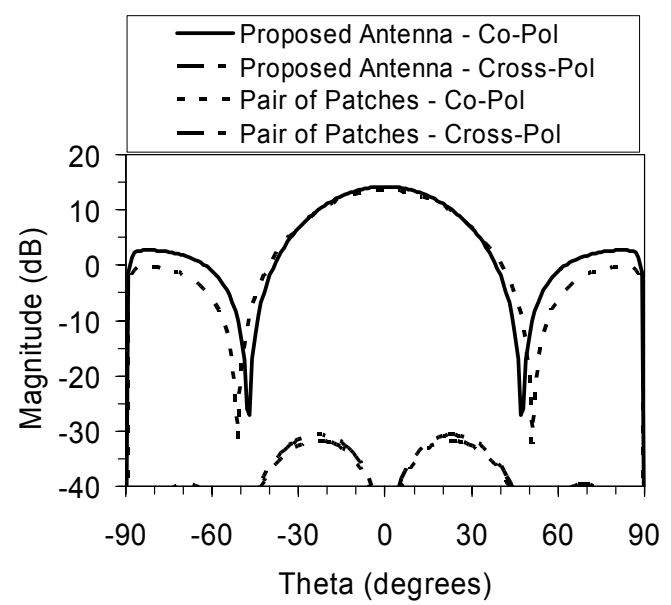

Fig 4. Calculated radiation patterns relative to isotropic of the proposed antenna and the pair of patches.

(a)

(b)
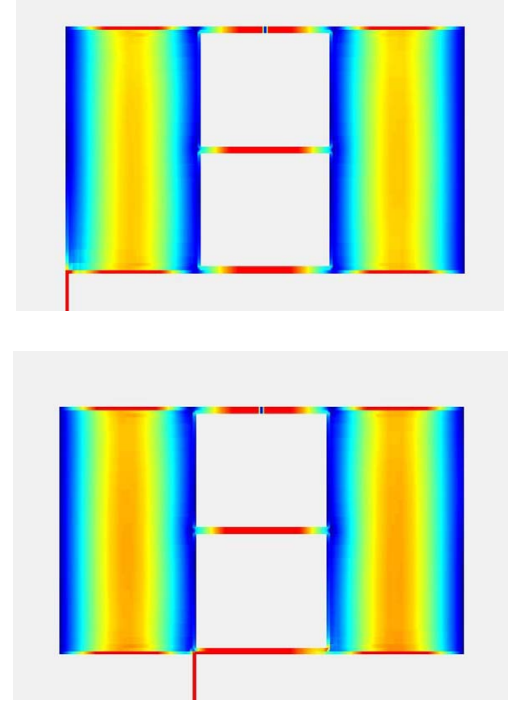

Fig 5. Calculated surface current density at $2.482 \mathrm{GHz}$ for excitation applied to: (a) port 1 , and (b) port 2 . The colour scale in both cases is identical.

\section{EXPERIMENT}

A test antenna was fabricated to demonstrate the feasibility of the antenna as well as being a reference antenna for eventual test of an active antenna. A photograph is shown in Fig 6 . It comprises the proposed 4-port antenna with 
dimensions and substrate as described in section III and a feeding network. The dc bias connection port was short-circuited to ground. The ports are fed using a transmission line and rat-race coupler: in essence, the circuit of Fig 1(b) but with the transistors replaced with short lengths of $1 \mathrm{~mm}$ wide microstriplines. Fig 7 shows the simulation results for this antenna along with experimental results obtained with a HP8753D vector network analyzer. It can be seen that the calculated and measured return loss frequency response are very similar. The out-of-band return loss is due to cable loss. Probing of the antenna confirmed that the antenna indeed radiated in the pass-band centred at $2.47 \mathrm{GHz}$.

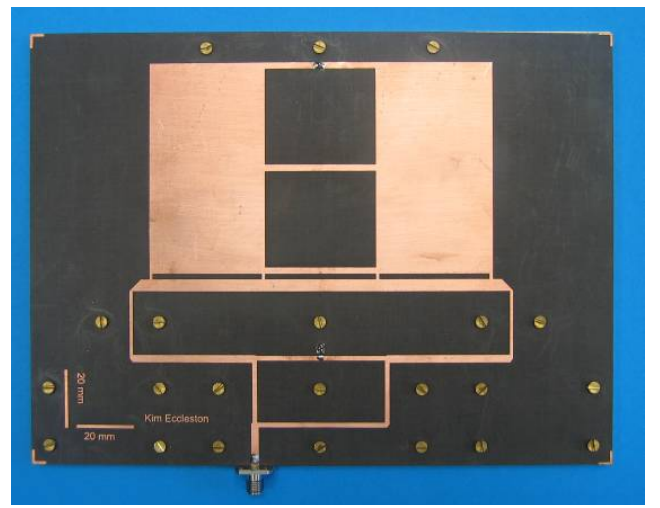

Fig. 6. Photograph of test antenna.

\section{CONCLUSION}

In this paper we have proposed a 4-port microstrip antenna that combines power on the antenna prior to radiation. The antenna has four radiating edges that are spaced approximately half-wavelength. The radiation pattern is similar to a 2-element array of half-wave patches. The ports are located along one of its edges allowing easy connection of microwave transistors and means that it can be integrated in a dual-fed distributed amplifier. The load presented at each port is nearly identical which ensures efficient transistor operation and equal utilization of the transistors. Drain bias for the transistors can be conveniently fed using the antenna. Simulation results were used to confirm the feasibility of the approach. Reflection coefficient measurements of a test antenna were used to valid the simulations.

\section{ACKNOWLEDGEMENT}

The author would like to thank the assistance of Mr Randy Hampton and Mr Ron Battersby for their kind assistance in fabricating the antenna.

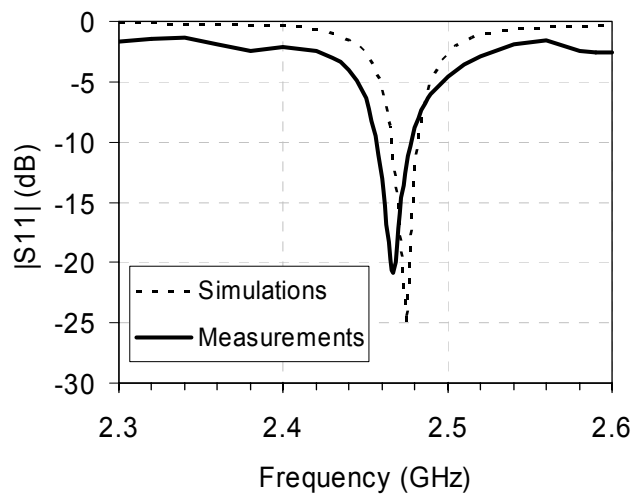

Fig 7. Return loss of the test antenna.

\section{REFERENCES}

[1] K. Chang, R. A. York, P. S. Hall and T. Itoh "Active Integrated Antennas," IEEE Trans. on Microwave Theory and Techniques, 50(3), pp 937 944, March 2002.

[2] W. R. Deal, V. Radisic, Y. Qian and T. Itoh, "Integrated-Antenna Push-Pull Power Amplifiers", IEEE Trans. on Microwave Theory and Techniques, 47(8), pp 1418 - 1425, Aug 1999.

[3] W. Wang and Y. P. Zhang, "0.18- $\mu$ m CMOS PushPull Power Amplifier With Antenna in IC Package", IEEE Microwave and Wireless Component Letters, 14(1), pp 13 - 15, Jan 2004.

[4] S. Gao and P. Gardiner, "Novel Integrated Antenna for LINC Power Amplifiers", 2002 IEEE Antennas and Propagation Symposium, pp 508 - 511.

[5] S. Gao and P. Gardiner, IEEE Trans. on Microwave Theory and Techniques, 53(3), pp 1083 - 1088, March 2005.

[6] K. W. Eccleston and S. Bommana, "Four-port Microstripline Antenna Integrated With a Distributed Amplifier", 2005 Asia Pacific Microwave Conference (APMC2005), 4-7 Dec 2005, Suzhou, China, pp833-836.

[7] K. W. Eccleston, "Compact Efficient Dual-Fed Distributed Power Amplifier", IEEE Transactions on Microwave Theory and Techniques, 53(3), March 2005, pp 825-831.

[8] C. A Balanis, Antenna Theory, Analysis and Design, $2^{\text {nd }}$ Ed., John Wiley and sons, 1997, p 732. 